\title{
A perturbation analysis of the flow of a Powell-Eyring fluid between coaxial cylinders
}
M. T. J. Farrugia ${ }^{1}$
J. J. Shepherd ${ }^{2}$
A. J. Stacey ${ }^{3}$

(Received 3 December 2010; revised 19 April 2011)

\begin{abstract}
The Powell-Eyring generalised viscous fluid model was developed to model the flow of viscous inelastic shear thinning fluids, such as polymer melts and suspensions of solids in non-Newtonian solvents. We consider the helical flow of a Powell-Eyring fluid between infinitely long coaxial cylinders. Such a flow is of relevance to a number of applications, including rheometry. We show that a perturbation approach to the flow problem yields simple explicit expressions for the fluid velocity field, which may then be applied to obtain approximations to other features of the flow.
\end{abstract}

\section{Contents}

\section{Introduction}

C258

2 Governing equations

C259

http://anziamj.austms.org.au/ojs/index.php/ANZIAMJ/article/view/3748 gives this article, (c) Austral. Mathematical Soc. 2011. Published June 27, 2011. ISSN 1446-8735. (Print two pages per sheet of paper.) Copies of this article must not be made otherwise available on the internet; instead link directly to this URL for this article. 
3 Perturbation analysis for flow in a narrow gap

4 Discussion

C268

References

C269

\section{Introduction}

When an incompressible viscous fluid flows in the gap between infinitely long coaxial cylinders, with an axial fluid velocity superimposed on a transverse rotational flow, the resulting fluid motion is termed a helical flow. Such flows are of interest in applications, particularly in modelling the action of a cup and bob rheometer modified to allow axial flow, in order to carry out measurements on slurries and other settling mixtures [1]. When the fluid is Newtonian, such helical flow is well understood. However, when the fluid is non-Newtonian, so that the constitutive equation linking stress and rate of shearing is nonlinear, the task of obtaining the velocity profile and associated fluid properties is considerably more difficult and numerical techniques are usually employed. While this problem was solved in general terms by Coleman and Noll [2], their analysis converted a nonlinear boundary value problem to a nonlinear algebraic one, leaving the details of the solution of either as an unsolved problem.

In view of the above rheometer application, one situation becomes of significance to the present analysis. In many applications, the inter-cylindrical gap is small, to minimise experimental side effects. In such cases, a perturbation procedure based on some normalised form of the inter-cylindrical gap as perturbation parameter is indicated to solve the nonlinear equations of motion. This approach has been used to analyse the helical flow of similar fluids [1].

We apply such a perturbation approach to the laminar helical flow of a PowellEyring fluid, a pseudo-plastic fluid model used to represent viscous inelastic 
shear thinning fluids $[3, \S 1.3]$, such as polymer melts and suspensions. This analysis will parallel and extend earlier work of Farrugia [4].

\section{Governing equations}

We consider steady helical flow of an incompressible viscous fluid in the infinitely long annular region described in cylindrical polar coordinates $(r, \theta, z)$ by $R_{1} \leqslant r \leqslant R_{2}, 0 \leqslant \theta \leqslant 2 \pi,-\infty \leqslant z \leqslant \infty$. The inner cylindrical surface $r=R_{1}$ is given a constant angular velocity $\Omega>0$, the outer cylinder $r=R_{2}$ is held stationary, and a given axial flow rate $\mathrm{Q}>0$ is imposed.

The equations of motion for such a flow are well documented [2]. With the $z$-axis taken as vertically down and assuming all quantities exhibit symmetry about this axis, the velocity field which satisfies the equation of conservation of mass takes the form

$$
\left(u_{r}, u_{\theta}, u_{z}\right)=(0, r W(r), v(r)),
$$

for appropriate functions $V(r)$ and $W(r)$. One integration of the momentum equations yields differential equations for the functions $V(r)$ and $W(r)$ :

$$
H V^{\prime}(r)=\alpha r+\frac{\beta}{r}, \quad H W^{\prime}(r)=-\frac{M}{2 \pi r^{3}} .
$$

Here $\mathrm{H}$ is the fluid viscosity, $\mathrm{M}>0$ may be interpreted as the moment per unit length exerted on the inner cylinder $r=R_{1}$, and $\alpha$ and $\beta$ are constants of integration to be determined by the application of the boundary conditions at the cylinder walls.

The non-slip conditions on the inner and outer cylinders yield the boundary conditions

$$
\mathrm{V}\left(\mathrm{R}_{1}\right)=\mathrm{V}\left(\mathrm{R}_{2}\right)=0, \quad \mathrm{~W}\left(\mathrm{R}_{1}\right)=\Omega, \quad \mathrm{W}\left(\mathrm{R}_{2}\right)=0 .
$$


Eliminating $\mathrm{H}$ between the equations (1) yields the relationship

$$
V^{\prime}(r)=-2 \pi\left(\alpha r^{2}+\beta\right) r^{2} W^{\prime}(r) / M .
$$

Further, $\mathrm{V}(\mathrm{r})$ is related to the volume flow rate

$$
\mathrm{Q}=2 \pi \int_{\mathrm{R}_{1}}^{\mathrm{R}_{2}} r V(r) d r .
$$

We regard the flow rate $\mathrm{Q}$ as known, so that (4) becomes a constraint on $\mathrm{V}(\mathrm{r})$. The local rate of shearing, $K=\sqrt{\left[\left(\mathrm{rW}^{\prime}\right)^{2}+\mathrm{V}^{\prime 2}\right] / 2}$, in terms of the gradients of $\mathrm{V}$ and $\mathrm{W}$. The boundary conditions (2) imply that $W^{\prime}(r)<0$ in all $\mathrm{R}_{1}<\mathrm{r}<\mathrm{R}_{2} ;$ so that $\left|\mathrm{W}^{\prime}\right|=-\mathrm{W}^{\prime}$. This, with (3), converts $\mathrm{K}$ to

$$
K=-r W^{\prime} \phi(r, \alpha, \beta) / \sqrt{2}, \quad \text { where } \phi=\sqrt{1+(2 \pi / M)^{2} r^{2}\left(\alpha r^{2}+\beta\right)^{2}} .
$$

For generalised Newtonian fluids, the viscosity $\mathrm{H}$ is a function of the local rate of shearing $\mathrm{K}$; that is, $\mathrm{H}=\mathrm{H}(\mathrm{K})$. For the Powell-Eyring fluid, this is

$$
\mathrm{H}(\mathrm{K})=\mathrm{A}+\mathrm{B} \operatorname{arcsinh}(\mathrm{CK}) / \mathrm{K},
$$

where $\mathrm{A}, \mathrm{B}$ and $\mathrm{C}$ are positive constants.

Note that putting $B=0$ or $C=0$ in (6) gives $H(K)=A$, and the PowellEyring fluid reduces to a Newtonian fluid, of constant viscosity A. Further, $\mathrm{H}(\mathrm{K}) \rightarrow \mathrm{A}$, in the high shear limit as $\mathrm{K} \rightarrow \infty$, since $\operatorname{arcsinh}(\mathrm{CK})=\ln (2 \mathrm{CK})+$ $\mathcal{O}\left(\mathrm{K}^{-2}\right)$ as $\mathrm{K} \rightarrow \infty$. Thus, for high shear rates, this fluid is approximately Newtonian.

Applying (5) and (6) to the second of (1) gives

$$
\operatorname{arcsinh}\left(-\frac{C}{\sqrt{2}} W^{\prime} r \phi\right)=\frac{M \phi}{2 \sqrt{2} B \pi r^{2}}+\frac{A W^{\prime} r \phi}{\sqrt{2} B},
$$

a first order nonlinear differential equation for $W$ that can be solved, in principle, subject to one of the boundary conditions (2). 
We now render the problem (1)-(7) dimensionless, with characteristic radial distance $R$, and dimensionless parameter $\varepsilon$ measuring the inter-cylindrical gap width:

$$
R=\left(R_{1}+R_{2}\right) / 2, \quad \varepsilon=\left(R_{2}-R_{1}\right) /\left(R_{1}+R_{2}\right),
$$

while the dimensionless constants $\mathrm{a}, \mathrm{b}, \sigma$ and radial variable $s$ are defined by

$$
a=-2 \pi \varepsilon R^{3} \alpha / M, \quad b=2 \pi \varepsilon R \beta / M, \quad \sigma^{2}=b / a, \quad r=R s,
$$

respectively. Dimensionless forms of $\mathrm{V}, \mathrm{W}, \mathrm{K}$ and $\mathrm{H}$, denoted by $v(\mathrm{~s}), \boldsymbol{w}(\mathrm{s})$, $\kappa(s)$ and $\eta(s)$, are defined by

$$
v=\Delta \mathrm{V}(\mathrm{Rs}), \quad w=\Delta \mathrm{RW}(\mathrm{Rs}), \quad \mathrm{k}=\sqrt{2} \Delta \mathrm{RK}(\mathrm{Rs}), \quad \eta=\mathrm{H}(\mathrm{K}(\mathrm{Rs})) / \mathrm{A},
$$

where $\Delta=2 \pi A R /(M \varepsilon)$, while dimensionless parameters $\gamma, \delta$, q and $\omega$ are defined by

$$
\gamma=\frac{C M \varepsilon}{2 \sqrt{2} \pi A R^{2}}, \quad \delta=\frac{2 \sqrt{2} \pi B R^{2}}{M \varepsilon}, \quad q=\frac{2 A Q}{M \varepsilon^{2} R}, \quad \omega=\frac{2 \pi A R^{2} \Omega}{M \varepsilon} .
$$

Note that $\gamma$ and $\delta$ derive from the fluid parameters $A, B$ and $C$, while $q$ and $\omega$ are related to the axial flow rate $Q$ and inner cylinder angular velocity $\Omega$.

In terms of the dimensionless quantities above, the flow problem in the intercylindrical gap, $s_{1}=1-\varepsilon \leqslant s \leqslant 1+\varepsilon=s_{2}$, now comprises the nonlinear differential equation for $w(s)$,

$$
\operatorname{arcsinh}\left(-\gamma s \psi(s, a, \sigma) w^{\prime}\right)=\frac{s \psi(s, a, \sigma)}{\delta \varepsilon}\left(\varepsilon w^{\prime}+1 / s^{3}\right),
$$

where $\psi(s, a, \sigma)=\sqrt{1+(s a / \varepsilon)^{2}\left(s^{2}-\sigma^{2}\right)^{2}}$, with the linked equation for $v(s)$,

$$
v^{\prime}=a s^{2}\left(s^{2}-\sigma^{2}\right) w^{\prime} / \varepsilon,
$$

subject to the boundary conditions

$$
v\left(s_{1}\right)=v\left(s_{2}\right)=0, \quad w\left(s_{1}\right)=w, \quad w\left(s_{2}\right)=0 .
$$


Note that equations (10) imply the weaker conditions

$$
\int_{s_{1}}^{s_{2}} v^{\prime}(s) d s=0 \text { and } \int_{s_{1}}^{s_{2}} w^{\prime}(s) d s=-\omega
$$

The condition (4) becomes, on integrating by parts, and using (10) and (11),

$$
\int_{s_{1}}^{s_{2}}\left(s^{2}-\sigma^{2}\right) v^{\prime}(s) d s=-\varepsilon q
$$

The local rate of shearing, given by (5) and the constitutive equation (6), may also be converted to the dimensionless forms

$$
\kappa(s)=-s w^{\prime}(s) \psi(s, a, \sigma) \text { and } \eta(\kappa)=1+\delta \operatorname{arcsinh}(\gamma \kappa) / \kappa,
$$

respectively.

For given values of $\mathbf{a}, \sigma, \gamma, \delta, \varepsilon$ and $\mathbf{q}$, the differential equations (8) and (9) may be integrated subject to any pair of the boundary conditions (10) involving both $v$ and $w$, to obtain the functions $v(s)$ and $w(s)$. Imposition of the full set of conditions (10), together with the flow condition (12) yields a set of three nonlinear equations of the form

$$
f_{i}(a, \sigma, \gamma, \delta, \omega, q, \varepsilon)=0, \quad i=1,2,3 .
$$

In principle, any two of these may be solved for a and $\sigma$ in terms of the other quantities. Substitution of these values in the third equation then yields an equation of the form

$$
\mathrm{F}(\gamma, \delta, \omega, \mathbf{q}, \varepsilon)=0
$$

This equation defines a relationship linking $\gamma, \delta$ and $\omega$, with $\mathrm{q}$ and $\varepsilon$ present as parameters. Equivalently, an analogous relationship links $\Omega$ with $M$. This relationship, sometimes termed the Reiner-Riwlin equation, is fundamental to this flow for the given fluid [5, p.231]. If $\gamma$ and $\delta$ are assumed given (that is, the flow occurs under constant torque conditions - typical of rheometer 
applications), then the relationship (13) determines $\omega$ in terms of $\mathbf{q}$ and $\epsilon$. Thus, the quantities a and $\sigma$ may be viewed as functions of $\mathbf{q}$ and $\varepsilon$. Consequently, the functions $w$ and $v$ described above may be regarded as functions of $\mathrm{q}$ and $\epsilon$ as well as $s$.

In general, the nonlinearity of the component equations above renders this process well-nigh impossible. However, in one case of interest in applications, solutions may be obtained by approximate methods. For a large class of helical flows, the inter-cylindrical gap is small, and thus, so is $\varepsilon$ (typical values of $R_{1}=0.024, R_{2}=0.025$, give $\varepsilon=0.0204$ ), while the $\varepsilon$-dependence of $q$ may be predicted; so that perturbation methods based on $\varepsilon \rightarrow 0$ apply.

\section{Perturbation analysis for flow in a narrow gap}

We now employ a perturbation procedure based on the limit $\varepsilon \rightarrow 0$, typified by a small inter-cylindrical gap, to construct approximate expressions for $v(s)$ and $w(s)$ and the constants a and $\sigma$. We estimate the orders of terms in Equations (8)-(12) as $\varepsilon \rightarrow 0$, by first estimating such orders for the constants a, $\sigma, \gamma, \delta$ and $\omega$. To do this, we return to the dimensional equations (1) to (6). Given $\Omega$ is independent of $\varepsilon$, the boundary condition (2) implies that $W^{\prime}(r)$ is $\mathcal{O}\left(\varepsilon^{-1}\right)$ over the inter-cylindrical gap. With (5), this implies that $\mathrm{K}$ is $\mathcal{O}\left(\varepsilon^{-1}\right)$ there, and (6) gives the result that $\mathrm{H}(\mathrm{K})$ is $\mathcal{O}(1)$. Applying these estimates to the second of (1), we have that $M$ is $\mathcal{O}\left(\varepsilon^{-1}\right)$. These estimates of $W^{\prime}$ and $M$ would seem to make (3) imply that $V^{\prime}$ is $\mathcal{O}(1)$ throughout the gap. However, if we argue that an appropriate physically real estimate for $\mathrm{V}$ is to be $\mathcal{O}(1)$ in the gap, an estimate of $\mathrm{V}^{\prime}$ as $\mathcal{O}\left(\varepsilon^{-1}\right)$ seems suitable. On the other hand, we know, from the boundary condition (2) that $V^{\prime}=0$ at a point interior to the gap. This must come from $\alpha r^{2}+\beta$ vanishing there; and the only estimate that will give consistency between orders of magnitudes in each side of (3) is that $\alpha$ and $\beta$ are both $\mathcal{O}\left(\varepsilon^{-2}\right)$. This gives $\mathrm{V}$ 
as $\mathcal{O}(1)$ throughout the gap; and then (4) gives $\mathrm{Q}$ as $\mathcal{O}(\varepsilon)$.

The estimates obtained above then give $a, b, \sigma, \gamma, \delta$, q, and $\omega$ as $\mathcal{O}(1)$ constants, and $v(\mathrm{~s})$ and $w(\mathrm{~s})$ as $\mathcal{O}(1)$ functions throughout the inter-cylindrical gap, with $\boldsymbol{v}^{\prime}(\mathrm{s})$ and $\boldsymbol{w}^{\prime}(\mathrm{s})$ being $\mathcal{O}\left(\varepsilon^{-1}\right)$. Since $\mathrm{s}^{2}-\sigma^{2}$ is $\mathcal{O}(\varepsilon), \psi(\mathrm{s}, \mathrm{a}, \sigma)$ is $\mathcal{O}(1)$, while the orders in (9) balance.

The unknown quantities in this problem are the functions $w(s)$ and $v(s)$ and the constants $a$ and $\sigma$. We now propose that these be represented as perturbation expansions in terms of the (small) parameter $\varepsilon$ that, based on the estimates above, take the form

$$
\begin{aligned}
w^{\prime}(s) & =\varepsilon^{-1} w_{0}^{\prime}(s)+f_{1}(\varepsilon) w_{1}^{\prime}(s)+\cdots, \\
v^{\prime}(s) & =\varepsilon^{-1} v_{0}^{\prime}(s)+g_{1}(\varepsilon) v_{1}^{\prime}(s)+\cdots, \\
a & =a_{0}+v_{1}(\varepsilon) a_{1}+\cdots, \\
\sigma & =\sigma_{0}+\mu_{1}(\varepsilon) \sigma_{1}+\cdots,
\end{aligned}
$$

where $\varepsilon f_{1}(\varepsilon), \varepsilon g_{1}(\varepsilon), v_{1}(\varepsilon), \mu_{1}(\varepsilon) \rightarrow 0$ as $\varepsilon \rightarrow 0$. While we recognise that higher order terms are involved in (14)-(17), we only use the two term representations. Note that (14) and (15) define expansions for the derivatives of $w$ and $v$ in terms of derivatives of the coefficient functions $w_{0}, w_{1}, \ldots$ and $v_{0}, v_{1}, \ldots$

In the analysis below we, by substituting (14)-(17) into the equations (8)-(9) and (11)-(12) and seeking a balance of orders of terms as $\varepsilon \rightarrow 0$, obtain expressions for the constants $f_{1}(\varepsilon), g_{1}(\varepsilon), v_{1}(\varepsilon)$ and $\mu_{1}(\varepsilon)$, as well as the functions $w_{0}^{\prime}(\mathrm{s}), w_{1}^{\prime}(\mathrm{s}), v_{0}^{\prime}(\mathrm{s})$ and $v_{1}^{\prime}(\mathrm{s})$. Integration of these last, subject to the boundary conditions (10) at the outer boundary $s=s_{2}$, then yields expressions for $w_{0}(s), w_{1}(s), v_{0}(s)$ and $v_{1}(s)$, and hence, via (14) and (15), two-term approximations to $w(s)$ and $v(s)$ that satisfy the boundary conditions (10) at $s=s_{2}$.

Substituting the expansions (14)-(17) into the differential equation (8) and considering terms as $\varepsilon \rightarrow 0$, we have the leading order term on the left-hand 
side of (8) as

$$
\operatorname{arcsinh}\left(-\gamma s \psi_{0} w_{0}^{\prime} / \varepsilon\right)
$$

where $\psi_{0}=\psi\left(s, a_{0}, \sigma_{0}\right)$, while the right hand side is

$$
\frac{s \psi_{0}}{\delta \varepsilon}\left(w_{0}^{\prime}+1 / s^{3}\right) \text {. }
$$

Since $\operatorname{arcsinh}(x)=\ln (2 x)+\mathcal{O}\left(x^{-2}\right)$ as $x \rightarrow \infty$, we deduce that $(18)$ is $\mathcal{O}(\ln \varepsilon)$, while $(19)$ is $\mathcal{O}\left(\varepsilon^{-1}\right)$. Since there can be no match between these, we choose

$$
w_{0}^{\prime}+1 / s^{3}=0,
$$

and expect that (18) matches the right hand side of (8) at a higher level.

Solving (20), subject to the boundary conditions (10) at $s=s_{2}$, we then get

$$
w_{0}=\left(1 / s^{2}-1 / s_{2}^{2}\right) / 2 \text {. }
$$

Substitution of the expansion above into (9) and using (20) gives the leading order term as

$$
\varepsilon v_{0}^{\prime}=-a_{0}\left(s^{2}-\sigma_{0}^{2}\right) / s .
$$

This gives the leading order term of (15); and substituting this into the first equation of (11) and (12) and taking leading order terms gives

$$
\sigma_{0}=1, \quad a_{0}=3 q / 8,
$$

so that

$$
v_{0}^{\prime}=-\frac{3 q}{8 \varepsilon}\left(s-\frac{1}{s}\right) .
$$

Integrating subject to the outer boundary condition (10) gives

$$
v_{0}=\frac{3 q}{8 \varepsilon}\left\{\frac{1}{2}\left(s_{2}^{2}-s^{2}\right)-\ln \left(s_{2} / s\right)\right\} .
$$


Now consider the second (first order correction) terms in the expansions (14)(17). From (20) and (14), $\mathfrak{w}^{\prime}$ is $\mathcal{O}\left(\varepsilon^{-1}\right)$; so that, on substituting (14) into (8) and expanding for large arguments, we obtain (8) as

$$
\begin{aligned}
\operatorname{arcsinh}\left(-\gamma s \psi w^{\prime}\right) & =\ln \left(-2 \gamma s \psi_{0} w_{0}^{\prime} / \varepsilon\right)+\cdots \\
& =s \psi_{0} f_{1}(\varepsilon) w_{1}^{\prime} / \delta+\cdots
\end{aligned}
$$

Choosing

$$
f_{1}(\varepsilon)=\ln (\varepsilon)
$$

allows both both sides to be of matching order as $\varepsilon \rightarrow 0$, so that

$$
w_{1}^{\prime}=\frac{\delta}{s \psi_{0} \ln (\varepsilon)} \ln \left(\frac{2 \gamma \psi_{0}}{\varepsilon s^{2}}\right)
$$

and applying (10) at $s=s_{2}$ gives

$$
w_{1}(s)=-\frac{\delta}{\ln (\varepsilon)} \int_{s}^{s_{2}} \frac{1}{u \psi_{0}} \ln \left(\frac{2 \gamma \psi_{0}}{\varepsilon u^{2}}\right) d u
$$

Substituting (14)-(17) into (9), and taking leading order terms gives

$$
g_{1}(\varepsilon) v_{1}^{\prime}=\frac{a_{0} s^{2}}{\varepsilon}\left(s^{2}-\sigma_{0}^{2}\right) f_{1}(\varepsilon) w_{1}^{\prime}-\frac{v_{1}(\varepsilon) a_{1}}{\varepsilon^{2} s}\left(s^{2}-\sigma_{0}^{2}\right) .
$$

Noting (21), we see that these terms may be matched if we choose

$$
g_{1}(\varepsilon)=\ln (\varepsilon), \quad v_{1}(\varepsilon)=\varepsilon \ln (\varepsilon)
$$

and then

$$
v_{1}^{\prime}=\left(\frac{3 q}{8 \varepsilon} s^{2} w_{1}^{\prime}-\frac{a_{1}}{\varepsilon s}\right)\left(s^{2}-1\right) .
$$

Applying (23) to the first of (11) gives, on incorporating (22),

$$
a_{1}=-\frac{9 q \delta}{\varepsilon^{3} \ln (\varepsilon)} \int_{s_{1}}^{s_{2}} \frac{s\left(s^{2}-1\right)}{\psi_{0}} \ln \left(\frac{2 \gamma \psi_{0}}{\varepsilon s^{2}}\right) d s
$$


an expression for $a_{1}$ which can be evaluated numerically. Integrating (23) subject to the boundary conditions at $s=s_{2}$ gives

$$
v_{1}(s)=-\int_{s}^{s_{2}}\left(\frac{39}{8 \varepsilon} u^{2} w_{1}^{\prime}(u)-\frac{a_{1}}{\varepsilon u}\right)\left(u^{2}-1\right) d u,
$$

where $\boldsymbol{w}_{1}^{\prime}$ and $\boldsymbol{a}_{1}$ are given by (22) and (24) respectively.

Applying (9) to the first of (11), substituting the expansions for $\sigma$ and $w^{\prime}$ into the result and using the values for $\sigma_{0}, w_{0}^{\prime}$ and $w_{1}^{\prime}$ obtained above leads, on considering the remaining leading order terms, to the condition

$$
\ln (\varepsilon) \int_{s_{1}}^{s_{2}} s^{2}\left(s^{2}-1\right) w_{1}^{\prime} d s=-\frac{2 \mu_{1}(\varepsilon) \sigma_{1}}{\varepsilon} \int_{s_{1}}^{s_{2}} s^{-1} d s
$$

and a balance of orders as $\varepsilon \rightarrow 0$ is achieved by choosing

$$
\mu_{1}(\varepsilon)=\varepsilon^{3} \ln (\varepsilon) .
$$

Then, (25) gives, on using (22) and (24),

$$
\sigma_{1}=a_{1} /(36 q),
$$

which gives $\sigma_{1}$ in terms of $a_{1}$.

We thus arrive at the two term approximations

$$
\begin{aligned}
w(s) & =\varepsilon^{-1} w_{0}(s)+\ln (\varepsilon) w_{1}(s)+\cdots \\
v(s) & =\varepsilon^{-1} v_{0}(s)+\ln (\varepsilon) v_{1}(s)+\cdots
\end{aligned}
$$

These expansions satisfy the outer boundary condition (10) at $s=s_{2}$, to the level of accuracy considered. Evaluation of the right hand side of (27) at $s=s_{1}$ and careful estimation of the two terms shows their sum to be $\mathcal{O}\left(\varepsilon^{2} \ln (\varepsilon)\right)$; that is, this approximation satisfies the first of (10), the inner boundary condition, to the level of approximation considered.

Applying the third of (10) to (26) gives

$$
\omega=\varepsilon^{-1} w_{0}\left(s_{1}\right)+\ln (\varepsilon) w_{1}\left(s_{1}\right)+\cdots,
$$



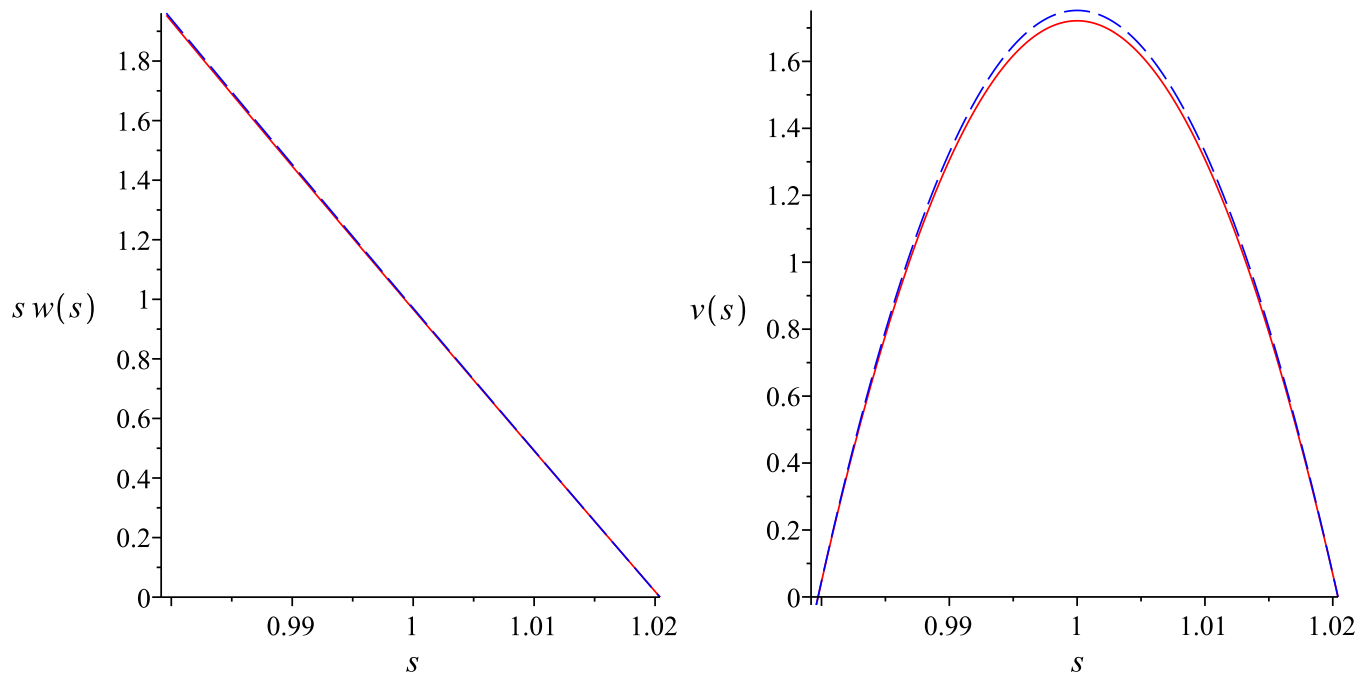

Figure 1: Radial (left) and axial (right) velocity profiles, for $A=1.2$, $\mathrm{B}=0.8, \mathrm{C}=0.9, \mathrm{Q}=0.0001, \mathrm{M}=5, \mathrm{R}_{1}=0.024$ and $\mathrm{R}_{2}=0.025$. Dashed lines show only the first term of the expansions.

which is a two term approximate relationship linking $\omega$ and the parameters $\varepsilon$, $\delta, \gamma$ and $\mathrm{q}$. This is an approximate form of the Reiner-Riwlin equation for this fluid and flow.

\section{Discussion}

The expressions (26)-(27) are explicit, readily evaluated approximations to the functions $w(s)$ and $v(s)$ and the constants a and $\sigma$ for small $\varepsilon$. Thus they may be used to obtain approximate expressions for the significant features of this helical flow. In particular, they may be used to represent the fluid velocity field $(s w(s), v(s))$. This is shown in Figure 1. It is apparent that the second term in (26) makes little difference to $s w(s)$; but for $v(s)$, there is some overall reduction, arising from the non-Newtonian nature of this fluid. 
These approximations may also be used to obtain approximate expressions for the viscosity profile $\eta(\kappa)$ and the rate of shearing, $\kappa$, as functions of $s$.

\section{References}

[1] C. Chiera, H. J. Connell and J. J. Shepherd, Perturbation analysis of the helical flow of non-Newtonian fluids with application to a recirculating coaxial cylinder rheometer, Mathematical and Computer Modelling, 18, No. 10: 131-140, 1993. C258

[2] B. D. Coleman and W. Noll, Helical flow of General Fluids, J. App. Phys., 10: 1508-1512, 1959. C258, C259

[3] R. I. Tanner, Engineering Rheology, Oxford University Press, Oxford, 1988. C259

[4] M. T. J. Farrugia, A Perturbation Analysis of the Flow of a Powell-Eyring Fluid between Coaxial Cylinders, Honours Thesis, Mathematics Department, RMIT, 1999. C259

[5] R. B. Bird, R. C. Armstrong and D. Hassager, Dynamics of Polymetric liquids, Vol. 1, John Wiley and Sons, 1987. C262

\section{Author addresses}

1. M. T. J. Farrugia, School of Mathematical and Geospatial Sciences, RMIT University, Melbourne, Australia.

2. J. J. Shepherd, School of Mathematical and Geospatial Sciences, RMIT University, Melbourne, Australia. mailto: jshep@rmit.edu.au 
3. A. J. Stacey, School of Mathematical and Geospatial Sciences, RMIT University, Melbourne, Australia. 